\title{
44353 - ADRENALINE DOES NOT INFLUENCE PCEA MEPERIDINE USE AFTER THORACOTOMY
}

\author{
Calvin Thompson, The Ottawa Hospital - General Campus, Ottawa, ON, Canada; \\ Gregory L Bryson, The Ottawa Hospital - Civic Campus; \\ Gagne Sylvain, The Ottawa Hospital - General Campus; \\ LJ Byford, The Ottawa Hospital - Civic Campus; \\ M Kattan, The Ottawa Hospital - General Campus; \\ J Penning, The Ottawa Hospital - Civic Campus;
}

INTRODUCTION: Patient controlled epidural analgesia (PCEA) with meperidine provides effective analgesia following thoracotomy. Accumulation of normeperidine, a meperidine metabolite with neuroexcitatory effects, has lead to recommendations to limit the use of meperidine postoperatively(1). The consumption and systemic levels of epidurally administered local anesthetics and opioids are reduced by the addition of adrenaline to the analgesic solution(2). The purpose of this study was to determine if the addition of adrenaline to PCEA meperidine was associated with decreased meperidine consumption, decreased serum normeperidine levels, and improved analgesia following thoracotomy.

METHODS: Following research ethics approval consenting patients were randomly assigned to either PCEA meperidine $2 \mathrm{mg} / \mathrm{ml}$ analgesia with adrenaline $2 \mathrm{mcg} / \mathrm{ml}$ (Group MAD) or PCEA meperidine $2 \mathrm{mg} / \mathrm{ml}$ plain (group MEP). All patients received a standardized anesthetic and similar perioperative care. Visual analogue pain scores (at rest and with activity), Quality of Recovery scores, and side effects were documented 6, 24, and 48 hours postoperatively. Serum levels of meperidine and normeperidine were assessed using tandem gas chromatography - mass spectrometry.

RESULTS: A total of 46 patients completed the study protocol. Patient characteristics and analgesia outcomes are shown in Table 1. Cumulative meperidine consumption at 48 hours was $601 \pm 211$ vs $580 \pm 211$ in MAD and MEP groups respectively; $(\mathrm{p}=0.744)$ Serum meperidine levels at 48 hours were $0.23 \pm 0.17$ vs $0.19 \pm 0.14$ in MAD and MEP groups respectively; $(\mathrm{p}=0.400)$. Serum normeperidine was not detected in any sample. 
TABLE 1. PATIENT CHARACTERISTICS AND PAIN OUTCOMES

\begin{tabular}{|l|c|c|c|}
\hline & $\begin{array}{c}\text { MAD } \\
(\mathbf{n = 2 2})\end{array}$ & $\begin{array}{c}\text { MEP } \\
(\mathbf{n = 2 4 )}\end{array}$ & P value \\
\hline Age (years) & $63.4 \pm 8.1$ & $60.0 \pm 8.7$ & 0.166 \\
\hline Height $(\mathrm{cm})$ & $164.5 \pm 8.6$ & $168.0 \pm 9.6$ & 0.196 \\
\hline Weight $(\mathrm{kg})$ & $70.5 \pm 14.4$ & $75.8 \pm 13.8$ & 0.209 \\
\hline Creatinine Clearance (ml/min) & $80.7 \pm 23.3$ & $88.3 \pm 24.9$ & 0.292 \\
\hline Pain (dynamic) & & & \\
\hline 6 hr post-op & $4.1 \pm 2.2$ & $4.4 \pm 2.4$ & 0.609 \\
\hline 24 hr post-op & $3.6 \pm 2.4$ & $3.5 \pm 2.6$ & 0.924 \\
\hline 48 hr post-op & $3.7 \pm 2.8$ & $4.1 \pm 2.9$ & 0.621 \\
\hline Quality of Recovery & & & \\
\hline 6 hrs & $13[7-14]$ & $13[10-15]$ & 0.625 \\
\hline 24 hrs & $13[9-14]$ & $13[9-14]$ & 0.620 \\
\hline 48 hrs & $13[8-18]$ & $14[7-18]$ & 0.093 \\
\hline Adverse Effects & & & \\
\hline Nausea & $13(59)$ & $14(58)$ & 0.958 \\
\hline Pruritus & $9(41)$ & $4(17)$ & 0.068 \\
\hline Respiratory Depression $(\mathrm{RR}<8)$ & $1(5)$ & $1(4)$ & 0.950 \\
\hline Hypotension (BP <90 mmHg) & $8(36)$ & $8(33)$ & 0.829 \\
\hline
\end{tabular}

Data expressed as mean $\pm \mathrm{SD}$, median [interguartile, range], or $\mathrm{n}(\%)$

DISCUSSION: The addition of adrenaline did not influence PCEA meperidine consumption, analgesia outcomes, or quality of recovery. Normeperidine did not accumulate in patients of either study group during the 48 hour study period. PCEA meperidine, with or without adrenaline, provides effective post-thoracotomy analgesia in selected patients.

REFERENCES: 1. http://www.jcaho.org. 2. Acta Anaesthesiol Scand 1998; 42(8):897-909. 